\title{
Improve information retrieval and e-learning using mobile agent based on semantic web technology
}

\author{
Maged Elazony, Momtaz Al-Kholy, Ahmed Khalifa, Sayed Nouh, \\ Mohamed Hussein, Gamal Tharwat \\ Azhar University, Faculty of Engineering, Computers and Systems Engineering \\ Department
}

\begin{abstract}
The Web-based education and E-Learning has become a very important branch of new educational technology. E-learning and Web-based courses offer advantages for learners by making access to resources and learning objects very fast, just-in-time and relevance, at any time or place. Web based Learning Management Systems should focus on how to satisfy the e-learners needs and it may advise a learner with most suitable resources and learning objects. But Because of many limitations using web 2.0 for creating E-learning management system, now-a-days we use Web 3.0 which is known as Semantic web. It is a platform to represent E-learning management system that recovers the limitations of Web 2.0.In this paper we present "improve information retrieval and e-learning using mobile agent based on semantic web technology". This paper focuses on design and implementation of knowledge-based industrial reusable, interactive, web-based training activities at the sea ports and logistics sector and use e-learning system and semantic web to deliver the learning objects to learners in an interactive, adaptive and flexible manner. We use semantic web and mobile agent to improve Library and courses Search. The architecture presented in this paper is considered an adaptation model that converts from syntactic search to semantic search. We apply the training at Damietta port in Egypt as a real-world case study. we present one of possible applications of mobile agent technology based on semantic web to management of Web Services, this model improve the information retrieval and E-learning system.
\end{abstract}

\section{Keywords}

Semantic web, ontology, mobility, intelligent agent, OWL, RDF, E-Learning, Mobile agent

\section{Introduction}

Traditional methods of search engines are not effective during the recovery of search results that may not be the user's needs. Traditional search engines cannot understand the text of the user and his interests because they rely on a set of keywords that are inserted by the users -for syntactic search- to get the desired results with a different indicators lead to incorrect results, irrelevant or no results to all users when searching.

The World Wide Web was not built for machine consumption it built for human consumption, although everything on the Web is not Machine-understandable, its machine-readable . the Semantic Web to express information in a machine interpretable, precise form, ready for software agents to share, process, and reuse it, as well as to understand what the terms describing the data 
mean. That would enable web-based applications to interoperate both on the semantic and syntactic level.

We need to represent the knowledge in different contexts on same web page and adapt to different users requirements and queries. To achieve that, we need to add new technologies that help machines to understand these documents. These technologies are the semantic web .Semantic Web can help search engines deliver better search results. In the traditional Web environment, each Web page only provides information for computers to display the page, not to understand it; the page is just intended for human eyes. Therefore, traditional search engines are forced to do keyword matching only. Without any semantics embedded in the page, the user gets quite a lot of irrelevant results. To solve this problem, we can extend the traditional Web by adding semantic data to it.

We can construct a vocabulary set that contains (all) the important words (concepts, classes) for a given domain, and the semantics and knowledge are coded into this set; more importantly, this set has to be constructed using some structured data.

We then markup a Web page by adding a pointer in its metadata section. This pointer points to the appropriate vocabulary set; this is how we add semantics to each page. When visiting a Web page, a mobile agent (or crawler in the search engine example) is able to see the link from its metadata section and follow it to retrieve the vocabulary set. As this set is constructed using structured data, the mobile agent is able to understand the vocabulary set. Also, as the given page is linked to this set, the mobile agent is able to understand what this page is all about.

The success of the Semantic Web depends on the integration, easy creation and use of semantic data .

The word semantic implies meaning. For the Semantic Web, semantic show that the meaning of data on the Web can be understand and discovered not just by people, but also by machine (computers). Most meaning on the Web today is inferred by people who read web pages and the labels of hyperlinks, and by other people who write specialized software to work with the data. The phrases "Semantic Web" stands for a vision, in which computers- software - as well as people can find, read, understand, and use data over the World Wide Web to accomplish useful goals for users .The Semantic Web is the abstract representation of data on the World Wide Web, based on the RDF standards and other standards to be defined.

We consider distributed Web service components that can be discovered in the Web based on semantic annotations, move to any target platform carried by mobile agents and perform their tasks locally, fast, and cooperatively.

The main point is that an agent can carry out tasks without human supervision. So, an agent is a computer system capable of autonomous action in some environment controlling its own internal state.

Agents are best for applications that are modular, decentralized, changeable, badly structured and complex. In fact, mobile agents will turn the web services into proactive entities working as peers 
International Journal of Web \& Semantic Technology (IJWesT) Vol.6, No.3, July 2015

to serve the user, representing and defending interests in a competitive world where services are negotiated and composed dynamically.

Mobile agents introduce unparalleled level of autonomy into future systems so that users can delegate high-level tasks in a generic manner. So Mobility of agents is an important property

- The rest of this paper is organized as follows. Section (2)introduces the traditional web and its limitations, section (3) Semantic Web Technologies ,section(4 )The Semantic Web Architecture, section (5) Mobile Agent (Mobility) and Semantic Web section (6) Case study - E-Learning Based On Semantic Web Technology and Mobile Agent. the paper is finally concluded in section (7).

\section{Traditional Web restriction}

"When you use a web browser to display a web page, it reads a file containing a linear sequence of characters, most of which is passed unchanged. Hidden in the original sequence, however, there are instructions which tell the browser how to display images, format text, link to other pages, and so on. These instructions are called "tags". This collection of tags - and their attributes - is called HTML (Hypertext Markup Language)".

HTML identifies the structure of documents for retrieval across the Internet using browser of the World Wide Web. It is the standard markup language used to create web pages, It was designed to create web pages and to display the data on these web pages. But doesn't focus on what the data is or how the data can be stored or transported in an effective and efficient way.

The content of the WWW may be classified into documents and data . Where data may be processed by machines to make them readable and can be handled by the human. And documents are everything readable by the human like reports and mails

Search engines are optimized to retrieve specific per-defined and precise specifications.

So that, the end users should know exactly what words to use and the search result for these words will be precise and accurate. It is assumed the end user knows the exact terms to investigate. This suitable in a basic retrieval model, but if the user does not know exactly what words to use in the search engines then the traditional search tools will not useful.

Today's World Wide Web (WWW) includes web applications, blogs, wikis, social networking sites, video sharing sites, hosted services it known as Web 2.0; it also includes E-learning Management system and Web-based courses.

Research works in the field of web based training and E-Learning are represented by a wide spectrum of applications, ranged from virtual classrooms to remote courses or distance learning. Web-based courses offer advantages for learners by making access to resources and learning objects very fast, just-in-time and relevance, at any time or place. 
International Journal of Web \& Semantic Technology (IJWesT) Vol.6, No.3, July 2015

Much progress has been made about the web and related technologies in the past two decades. Web 1.0 as a web of information, web 2.0 as a web of communication, web 3.0 as a web of cooperation and web 4.0 as a web of integration are introduced as four generation of the web since the advent of the web.

Because of many of limitations using web 2 for creating web based training and E-learning, we use Web 3.0 which is known as Semantic web. It is a platform to represent E-learning management system that recovers the limitations of Web 2.0.

The Semantic Web may be considered as an evolution to this WWW which aims to make all the information and application data on the internet universally shared and machine process able in a very efficient way. It is an intelligent web which can understand the information semantics and services on the Internet by applying technologies and enabling inference rules to increase users' satisfaction while searching the web content Motivations for inventing the Semantic Web are the limitations of the traditional Web Semantic Web provides solutions for these drawbacks by offering more efficient technologies such as XML, RDF and Ontology. The Semantic Web was introduced by Tim Berners- Lee who invented the traditional World Wide Web .

\section{Semantic Web Technologies Overview}

The Semantic Web is the extension of the WWW that allows people to share content beyond the boundaries of websites and applications. There are many different ideas about what the Semantic Web is. Berners-Lee, Hendler and Lassila (2001) define the Semantic Web, also known as Web 3.0, as "not a separate Web but an extension of the current one, in which information is given well-defined meaning, better enabling computers and people to work in cooperation".

Berners-Lee hopes that eventually computers will be able to use the information on the Web, not just present the information. "Machines become capable of analyzing all the data on the Web the content, links, and transactions between people and computers" .Based on his idea, the Semantic Web is a vision and is considered to be the next step in Web evolution. It is about having data as well as documents on the Web so that machines can process, transform, assemble, and even act on the data in useful ways. One of the great promises of the Semantic Web is flexibility in accessing and identifying information.

The Semantic Web is an extension of the current Web. It is constructed by linking current Web pages to a structured data set that indicates the semantics of this linked page. A smart agent, which is able to understand this structure data set, will then be able to conduct intelligent actions and make educated decisions on a global scale.

The Semantic Web takes the solution furthermore. It involves publishing in languages specifically designed for data: Resource Description Framework (RDF), Web Ontology Language (OWL), and Extensible Markup Language (XML) . In contrast with the simple HTML, the XML allows content creators to label information in a meaningful way (for example $<$ Car $><$ Model $>1981<$ Model $></$ Car $>$ ) but the machine still knows nothing about what is meant by this structure. The RDF then comes to represent the relationships between the data items and give more meaning for the XML labels. The OWL provides the semantics for the data syntax and 
solves some problems occurred such as "two databases may use different identifiers for the same concept", then the machine needs OWL to discover the data which have the same meaning. These expressive technologies enable the data to be represented in a machine readable structure and hence enable the machine applying inference rules on the data to obtain meaningful results to improve the search process.

briefly, the Semantic Web is supposed to make data located anywhere on the Web understandable and accessible, both by people and machines.

\section{The Semantic Web Architecture}

The term "Semantic Web" involve efforts to build a new World Wide Web architecture that enhances content with formal semantics, means the content is made suitable for machine only, as opposed to content that is only intended for human. This will enable automated agents to reason about Web content, and produce an intelligent response to unexpected situations. "Expressing meaning" is the main task of the Semantic Web. In order to achieve that objective several layers of representational structures are needed. They are presented in the Figure 1, among which the following layers are the basic ones .

The architecture can be divided into three parts which are :

- Knowledge representation (XML, XML schema and RDF, RDF schema layers).

- Ontology (Ontology vocabulary layer).

- Agents (Logic, Proof, Trust layers).

The important property of the Semantic Web architecture (i.e., common-shared-meaning and machine processable metadata), enabled by a set of suitable agents, establishes a powerful approach to satisfy the E-Learning requirements. The process is based on semantic querying and navigation through learning materials, enabled by the ontological background.

The Semantic Web can be exploited as a very suitable platform for implementing an E-learning system, because it provides all means for E-learning: ontology development, ontology-based annotation of learning materials, their composition in learning courses and active delivery of the learning materials through E-learning portals.

Tim Berners-Lee proposed four versions of Semantic Web architecture . Such versions describe the languages needed for data interoperability between applications in the form of layering architecture where each layer represents a language that provides services to the upper layer. However, layers described in such versions suffer from several deficiencies such as poor abstraction and rarely functional descriptions. Gerber . Avoided those deficiencies and designed a new architecture. Additional layer called "Rules" layer was added although its functionality is already embedded inside other layers. The authors in explored many layers compared to the Gerber's model. However, the overlapped functionalities among layers are more than overlapping in the Gerber's model and this increases the difficulty of the system engineering description and the layers integration. 
These versions describe the languages needed for data interoperability between applications in the form of layering architecture where each layer represents a language that provides services to the upper layer. As we are talking about architecture concept then there are some discrepancies and irregularities in Tim Berners-Lee's architectures considering the layered architecture evaluation criteria . The layered architecture evaluation criteria are clearly defined context, an appropriate level of abstraction, hiding of unnecessary implementation details, clearly defined functional layers, appropriate layering, and Modularity. These criteria are used as an evaluation for any layering architecture.

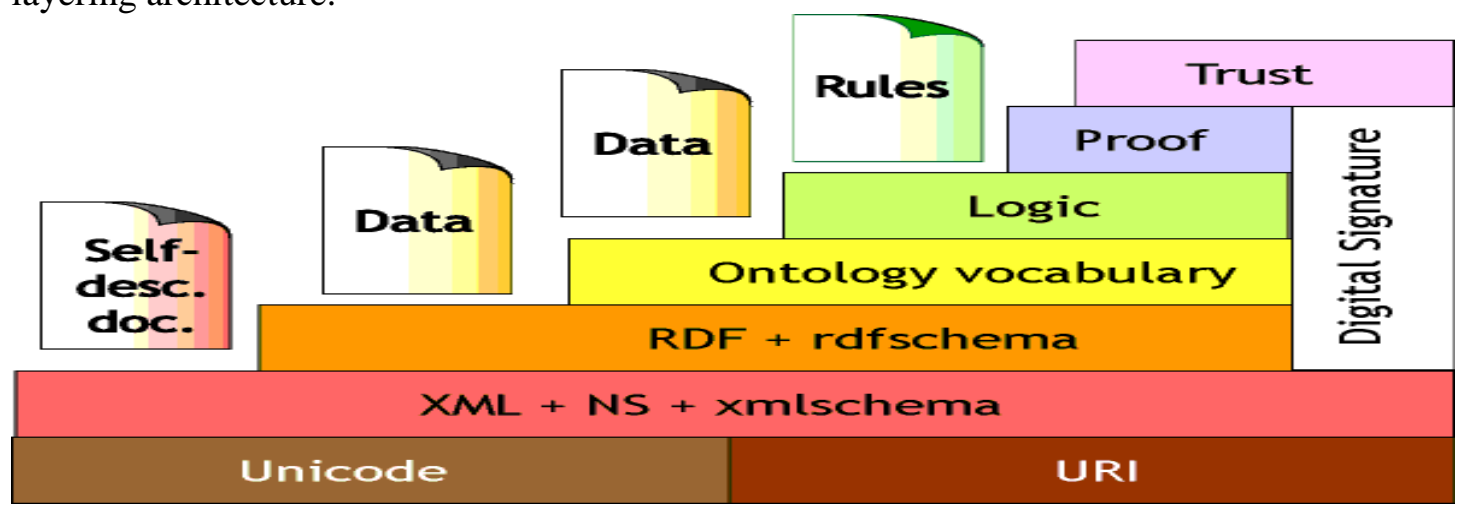

Figure 1-layers architecture of semantic web

Figure 1 shows the 9 layers architecture of semantic web in which the lowest layer starts from the bottom of the layer, followed by the highest layer, which is at the topmost position. The various layers and its challenges are described below.

\section{UNICODE}

Unicode is the basic universal number for every character, which works in multiple platforms. It is the basic notation, which is supported by top multinational companies like Motorola, IBM, Intel,etc. Unicode allows a single software, text or single character to be transported to other parts without corruption and re-engineering.

\section{URI (Uniform Resource Identifier)}

The URI is termed as Uniform Resource Identifier, which is a basic syntax for strings that is used to identify a resource. A resource is any physical or abstract things in which each item has an identifier. The URI consists of two types: First is Uniform Resource locators (URL) which identifies a resource and how it can be accessed, and the second part is Uniform Resource Names (URN)that is used to create a universal and persistent name about a resource in its namespace. This namespace dictates the syntax of URN identifier .

It is used to identify resources on the web, in which every resource in the WWW should be uniquely identified so we give it a URI. Resources could be anything such as a document, book, or video. There are different forms of the URIs. The most familiar form is the URL which is typed in the Web browser to locate its corresponding resource, so it has two functionalities which 
are identifying and locating the resources. There are other forms that only identify the resources but can't tell us their locations. Because the Web is too large to be controlled by only one person or organization, so creation of URIs is decentralized and anyone could create URI for her resources. It is clear that a problem of identifying the same resource with more than one URI may exist, but it is the cost of having such flexible and simple technique to identify resources on the Web.

\section{XML (Extensible Markup Language)}

The Extensible Markup Language (XML) is aW3C-recommended general-purpose markup language that supports a wide variety of applications. XML is also designed to be reasonably human-legible, and to this end, terseness was not considered essential in its structure . XML is evolved from simplified subset of Standard Generalized Markup Language (SGML). Its main task is to facilitate the sharing of data across different information systems, particularly systems connected via the Internet. XML is the simplest way to send the document across the web to its specific format. It allows users to edit or modify it and again transfer it. These document formats can include mark up also to enhance the meaning of the document XML is the primary and core foundation of the semantic web will be built on the top of XML. Scientifically, XML is built upon Unicode characters and URI's. The Unicode characters allow XML to be characterized using International characters :

\section{XML Schema}

XML Schema is a document definition language that enables you to develop XML documents into a Specific vocabulary and a specific hierarchical structure. The things you want to define in your language are element types, attribute types, and the composition of both into composite types (called complex types). XML Schema is different to database schema, which defines the column names and data types in database tables. XML Schema has been approved by W3C consortium in the year2001. XMLS allows the validation of instances to ensure the accuracy of field values and document structure at the time of creation. The accuracy of fields is checked against the type of the field; for example, a quantity typed as an integer or money typed as a decimal. The structure of a document is checked for things like legal element and attributes names, correct number of children, and required attributes. All XML documents should be checked for validity before they are transferred to another partner or system . There are different types of XML Schema Languages .

\section{XML Namespaces}

An XML namespace is the $\mathrm{W} 3 \mathrm{C}$ recommendation for providing uniquely named elements and all of its attributes in an XML instance. An instance of an XML contains element or attribute names from more than one vocabulary. If each vocabulary is given a namespace then the uncertainty or what is unexpected between identically named elements or attributes can be resolved. All the elements which are within a namespace must be in unique component . 
International Journal of Web \& Semantic Technology (IJWesT) Vol.6, No.3, July 2015

\section{RDF and RDF Schema}

Resource Description Framework (RDF) is a foundation of metadata processing. It provides interoperability between applications that exchange machine-understandable information on the Web . And it defines the relationship between the resources on the web.

RDF can represent by different syntaxes, one of the most popular syntax is the XML where the $\mathrm{RDF}$ based on this syntax is called RDF/XML model. The RDF statement is written in a triple form consisting of three parts which are the subject, the predicate and the object, so it seems like it is a natural phrase but its parts are URIs as they are resources on the Web.

A syntax (which is XML) representing this model is required to store instances of this model into machine-readable files and to communicate these instances among applications RDF imposes formal structure on XML to support the consistent representation of semantics .

The RDF and RDF Schema layer is located above the XML layer, which provides more functions and capabilities than in XML and XML schema. The Resource Description Framework is an XML based language that is used to describe resources. Such a resource is identified via a uniform resource locator (URL). As compared to XML documents that attach the metadata of the document, RDF captures the metadata of the externals of the document such as author, creation, date, etc. .

$\mathrm{RDF}$ is the machine processable language unlike XML which is human processable and it is not understandable by humans i.e. it knows what the machine is doing in the way it does. So that it can store smart information back on the Web.

RDF model is also called as triple, because it contains three parts viz. Subject, Predicate and Object. In Subject there is none of phrase that is the source of action. In Predicate, which is a part of triple edit the subject and includes the verb phrase? And in the object, it is a noun phrase that is the source of action by the verb .

RDFS or RDF Schema is a knowledge representation language, providing basic elements for the description of ontologies .

\section{Ontology Vocabulary}

The term "ontology" can be defined as an explicit specification of conceptualization . The conceptualization means modeling certain domain and the Ontology is used to describe important concepts of this domain, so it is the specification of this conceptualization. Ontology is the stage where the vocabularies related to a specific domain should be defined. It provides the capability to make analysis on the relationships between the vocabularies to discover problems such as the existence of two vocabularies of the same meaning. In this stage the relationships between vocabularies of a specific domain are created in hierarchal form by using the inheritance and classes concepts. Languages such as OWL (web ontology language) which may be considered as a syntactic extension for RDF/RDFS are provided at this stage. 
The main layer of semantic web architecture is Ontology vocabulary, which typically consists of hierarchical distribution of important concepts in a domain, along with descriptions of the properties of each concept.

OWL (Web Ontology Language): OWL is intended to be used when the information contained in documents needs to be processed by applications, as opposed to situations where the content only needs to be presented to humans. OWL can be used to explicitly represent the meaning of terms in vocabularies and the relationships between those terms. This representation of terms and their interrelationships is called ontology. OWL has more facilities for expressing meaning and semantics than XML, RDF, and RDF-S, and thus OWL goes beyond these languages in its ability to represent machine interpretable content on the Web. OWL has been designed to meet the requirements of RDF, RDFS, XML Schema .

\section{Logic Layer}

Logic layer in the semantic web is the universal language of monotonic logic. In the logic layer of semantic web architecture any rule can export the code but cannot import it. Any system in the logic layer can validate proofs. This layer functions on the basic principle of first order predicate logic, so the information is displayed accurately on the web.

\section{Proof}

In this layer, the final goal of semantic web is to create smarter content which could be understood by the machines. When the machine understood the content, some confirmation may come out of the content and new information will be produced. Unfortunately this layer has been not investigated enough and this lack of investigation is not yet enough considered to be a fateful problem.

\section{Trust}

This is the top most layer of the semantic web architecture in which the constancy of information should be subjectively evaluated by each information users. The trust layer in semantic web architecture is analyzed into four parts: The Information integration layer handles aggregation of information from different sources and adds provenance metadata in the information. The Repository Layer stores the aggregate information.

\section{Digital Signature}

Digital signatures layer run horizontal to the RDF family up through the layer proof and support the notion of trust. Developments of digital signatures are progressing, and could eventually help validate the integrity of metadata that an agent will use for reasoning and task completion. The main purpose of the digital signature is to digitally sign the document. Also encryption methods are used to run on digital signed documents to prevent against unauthorized access. 
International Journal of Web \& Semantic Technology (IJWesT) Vol.6, No.3, July 2015

\section{Mobile Agent (Mobility) and Semantic Web}

The utilized capacity of the server which provides a service is shortage of resources when it should serve a huge stream of online queries. This problem concerns a service provider, and can be solved by means of service reproduction and distribution of its copies to other servers in the Web. In this case it is possible to decrease the utilized capacity of the concrete source. That will also improve service discovery among a large amount of the services, which need mobility.

When a client of a service needs to use this service very often as such or as a part of a more complicated transaction involving several services. In this case we have frequent use of the network for service access. Besides, we cannot guarantee such important characteristics as Minimal service execution time, Guaranteed, permanent connection with service, Guaranty of confidentiality and secure private information exchange.

So the mobility of services plays an important role allowing "request" a service to a client side to serve locally.

\section{Case study - E-Learning Based on Semantic Web Technology and mobile agent}

The Semantic Web using as a backbone for eLearning. Foremost, the objectives are to ease the contribution of and the efficient access to information. But, in general, a Semantic Web-based learning process could be a a personalized (user customized), relevant (problem-dependent), and an active (context-sensitive) process these are prerequisites for realizing efficient learning We will describe the general model of solution in this section in details to understand how Library Search model retrieve these books.

\section{Library Search: the proposed Model}

The Library Search model is an example of semantic search engine based on semantic agent. The proposed model will effectively help users to find correct book based on the semantic query, which are implicitly and seamlessly injected into both user query and Library Search concepts stored in RDF, in contrast with traditional query which depends on keywords only, and not their meanings. The Library Search model is composed of two tiers, with each tier is responsible for specific task. The tier itself may be composed of multiple components. The Two tiers are structured as follows:

\section{The presentation tier}

This tier is the topmost level of the application. The presentation tier displays information related to Library search query and answers. This tier contact with other tiers by outputting results to the browser/client tier and all other tiers in the network. The Presentation layer has been implemented using J2EE technologies such as GWT, RPC and CSS. 


\section{The business logic tier}

This tier controls the application's functionality by performing detailed processing, handling transformation of input text, information exchange between the RDF data, the knowledge base (ontology) and the user interface. It Consisting of A group of agents that interact together to achieve a specific complete task related to a specific Library Search domain. An agent is a software component that has autonomy that provides an interoperable interface to other agents in the system, besides, it is adaptive, versatile and performs some diverse tasks (such as matching, ranking, parsing Ontology, reasoning over data etc...). This tier is decomposed into four main components described in the following subsections.

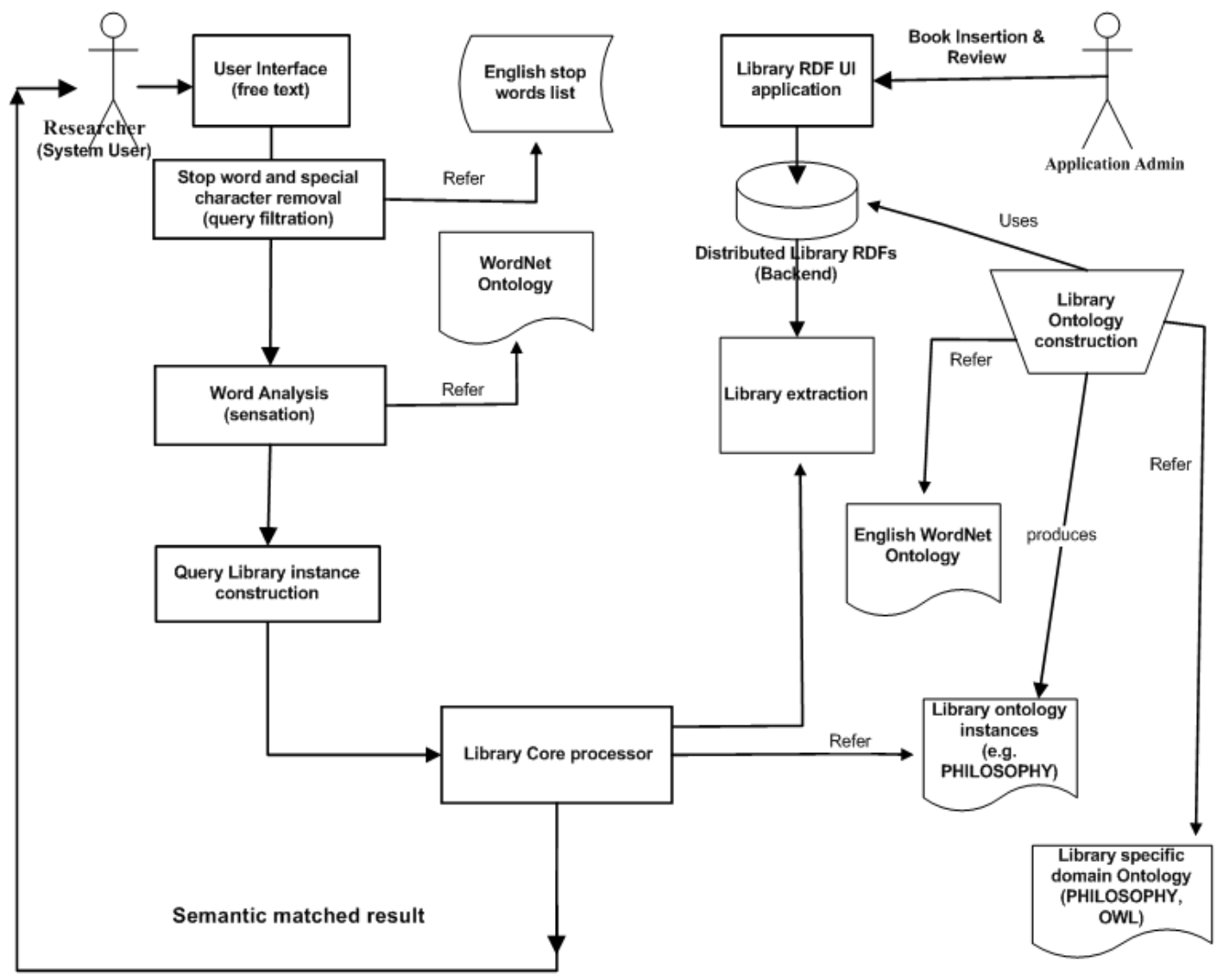

Figure 2 - Library Architecture details

\section{The stop words removal component (query filtration)}

When the user enters his query in the text area, he may enter other special characters such as punctuation marks. This component is responsible for filtering all special character and punctuation marks. the query words are separated by Stop words such as: if, with, on, from or the. Stop words are words which are filtered out prior to query processing. These words are 
eliminated by referring to a list contains stop words. Example of a query (Dynamics and Steady States in excitable mobile agent systems); after removing the stop words, the query becomes (Dynamics Steady States excitable mobile agent systems). The cause for eliminating the stop words is that these words do not add any crucial information and are not indexed.

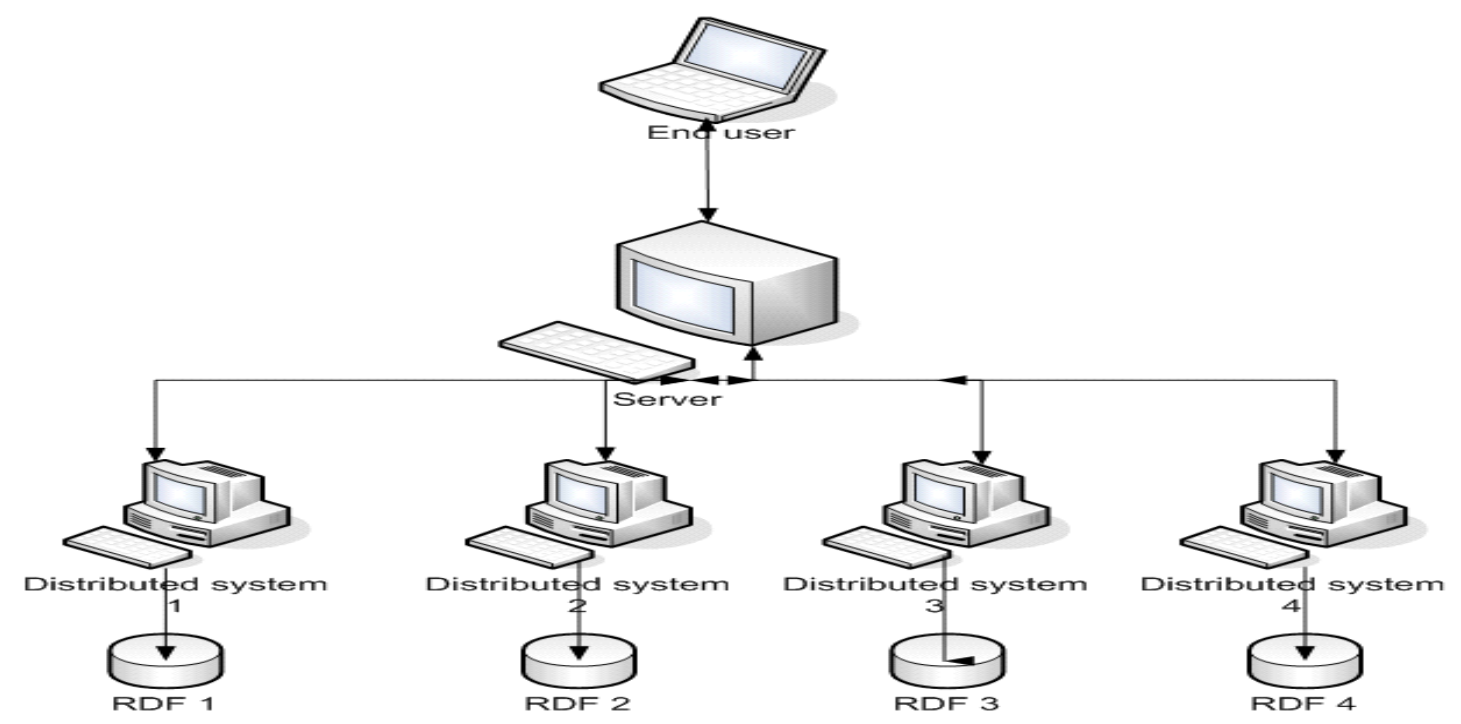

\section{The word analysis component (sensation)}

This component is the first semantic component that lists the words in the query and fetches their different meanings or references, by referring to predefined Word Net ontology for Library book terms and axioms used in any Library search domain. We mean by the term "different meaning", that a word may be lexically equivalent and consists of the same character, but with different meaning. As an example, the word (LOGIC), Which has meanings (Ontology) and (semantic). This component works as follow, first it tries to disambiguate word sense by using one of classification algorithms; if single definition is found, it processes this definition; otherwise it pops up a window proposing the word meaning to the user, with the facility to let the user select another meaning. Different WSD algorithms can be plugged here.

\section{The Query Library instance construction component}

As the query is normalized, another component begins the work of constructing anonymous domain instance that represents this query, in order to be matched against stored books names instance in knowledge base (RDFs). This component is responsible for this task.

\section{The Library core processor component}

This is the most important component in the model. It is the cornerstone of the system which performs multiple operations, including Library documents preparation, RDF indexation, instance ontology matching, Library ontology instance references, preparing the matched result to be sent to end user, performing some calculations to calculate the ratio of semantic distance between the 
International Journal of Web \& Semantic Technology (IJWesT) Vol.6, No.3, July 2015

question and the returned results and finally sorts the result according to their Interested data field.

\section{Used Technologies \\ GWT}

GWT Web Toolkit or Google Web Toolkit, is an open source set of tools that allows developers to maintain and create complex JavaScript front-end applications in Java. Other than a few native libraries, everything is Java source that can be built on any supported platform with the included GWT Ant build files. It is licensed under the Apache License version 2.0.

GWT emphasizes reusable approaches to common web development tasks, namely asynchronous remote procedure calls, history management, bookmarking, UI abstraction, internationalization, and cross-browser portability.

\section{GWT Advantages}

- A number of libraries are available for Google Web Toolkit, by Google and third parties.These extend GWT's features

- Open-source

- HTML Canvas support (subject to API changes)

- Support for full-featured Java debugging

- Dynamic and reusable UI components: programmers can use pre-designed classes to implement otherwise time-consuming dynamic behaviors, such as drag-and-drop or sophisticated visual tree structures

- Simple RPC mechanism

- Browser history management

- Support for using Google APIs in GWT applications (initially, support for Google Gears)

- GWT handles some cross-browser issues for the developer.

- Unit testing integration

- Support for Internationalization and localization

- The developers can mix handwritten JavaScript in the Java source code using the JavaScript Native Interface (JSNI)

- The developers can design and develop their application in a pure object-oriented fashion, since they're using Java (instead of JavaScript). Common JavaScript errors, such as typos and type mismatches, are caught at compile time

- The JavaScript that the GWT compiler generates can be tailored to be either un obfuscated or easier to understand or obfuscated and smaller to download.

\section{RPC}

In computer science, a remote procedure call (RPC) is an inter-process communication that allows a computer program to cause a subroutine or procedure to execute in another address space (commonly on another computer on a shared network) without the programmer explicitly coding the details for this remote interaction. That is, the programmer writes essentially the same code whether the subroutine is local to the executing program, or remote. When the software in 
International Journal of Web \& Semantic Technology (IJWesT) Vol.6, No.3, July 2015

question uses object-oriented principles, RPC is called remote invocation or remote method invocation.

\section{RPC Advantages}

- Enables the usage of the applications used in the distributed environment, not only in the local environment.

- The procedure calls preserves the business logics which is apt for the application.

- Process-oriented and thread oriented models supported by RPC

- Like the common communications between the portions of an application, the development of the procedures for the remote calls is quite general.

- Server independent

- The code re-writing / re-developing effort is minimized. The development of distributed systems is simple because it uses straightforward semantics and easier.

\section{CSS}

Cascading Style Sheets (CSS) is a style sheet language used for describing the look and formatting of a document written in a markup language. While most often used to style web pages and interfaces written in HTML and XHTML, the language can be applied to any kind of XML document, including plain XML, SVG and XUL. CSS is a cornerstone specification of the web and almost all web pages use CSS style sheets to describe their presentation.

CSS is designed primarily to enable the separation of document content from document presentation, including elements such as the layout, colors, and fonts.[1] This separation can improve content accessibility, provide more flexibility and control in the specification of presentation characteristics, enable multiple pages to share formatting, and reduce complexity and repetition in the structural content (such as by allowing for table less web design).

\section{CSS Advantages}

- Accessibility

- Bandwidth

- Separation of content from presentation

- Page reformatting

- Site-wide consistency

\section{AXIS}

Apache eXtensible Interaction System (Apache Axis) is an XML based Web service framework open source, It consists of a C++ implementation of the SOAP server a Java, and APIs and various utilities for deploying and generating Web service applications. Using Apache Axis, developers can create interoperable, distributed computing applications.

\section{AXIS Advantages}

- Easily retrieve the WSDL for a service. 
International Journal of Web \& Semantic Technology (IJWesT) Vol.6, No.3, July 2015

- Use Axis2's modular structure to easily add support for new recommendations as they emerge.

- Create implementation classes for both the server and client using WSDL.

- Receive and process SOAP messages.

- Create a Web service out of a plain Java class.

- Send and receive SOAP messages with attachments.

- Create or utilize a REST-based Web service.

- Send SOAP messages.

- Create or utilize services that take advantage of the WS-Security, WS-Reliable Messaging, WS-Addressing, WS-Coordination, and WS-Atomic Transaction recommendations

\section{Tomcat}

Apache Tomcat (or simply Tomcat, formerly also Jakarta Tomcat) is an open source web server and servlet container developed by the Apache Software Foundation (ASF). Tomcat implements the Java Servlet and the Java Server Pages (JSP) specifications from Sun Microsystems, and provides a "pure Java" HTTP web server environment for Java code to run in. In the simplest config Tomcat runs in a single operating system process. The process runs a Java virtual machine (JVM). Every single HTTP request from a browser to Tomcat is processed in the Tomcat process in a separate thread.

Apache Tomcat includes tools for configuration and management, but can also be configured by editing XML configuration files and this benefits has been used to make multi-services of the same application with different ports. This idea provides us with the same application functionality without duplications or creates more than virtual OS.

\section{Tomcat Advantages}

- Asynchronous Support.

- Application Performance/Reliability.

- Improved Logging.

- Extended Servlet API.

- Annotation Support.

- Dynamic Configuration.

- Simplified Embedding.

- Memory Leak Detection/Prevention.

- Improved Security.

We introduced the design and implementation of e-learning system using mobile agent based on semantic web technology offered by our web-based e-learning system, which is used by Damietta port customers and employees.

The important contributions of this paper are: 
International Journal of Web \& Semantic Technology (IJWesT) Vol.6, No.3, July 2015

- we present one of possible applications of mobile agent technology to management of semantic Web Services to improve Library and courses Search for E-learning Content Management System

- We have designed and implemented a successful information retrieval and e-learning system using mobile agent based on semantic web technology in Damietta port at Egypt.

- Improve information retrieval and e-learning system using mobile agent based on semantic web technology

- A user has access to the training and suitable learning objects from anywhere in the world using the Internet, breaking the limits of the number of participants on site and distance.

- The effective and efficient way to help trainer that dealing with ports to work smarter and operate more efficiently is through E-learning.

- Using semantic web and mobile agent to help user to Minimal service execution time, Guaranteed, permanent connection with service, and Guaranty of confidentiality and secure private information exchange.

\section{Conclusion}

This paper represents a part of our research work, proposes an adaptation model for information retrieval based on different methods and techniques from the following communities to achieve the model: ontology, Agent Technologies, Semantic Web and Web Services, Mobility, and Word Net ; it is applied on e-learning and Library search domain .This paper focuses on design and implementation of knowledge-based industrial reusable, interactive, web-based training activities at the port and logistics sector and use e-learning system and semantic web to deliver the learning objects to learners in an interactive, adaptive and flexible manner. We use semantic web and mobile agent to improve Library and courses Search. The architecture presented in this paper is considered an adaptation model that converts from syntactic search to semantic search. Sentences of Library Search are annotated with ontology propositions that recognize an instance of their content. Distributing ontology-based Information extraction to agents enables parallel processing and eases tracking of decisions and their explanation to users. An application example for the proposed system is introduced to show the advantages of using ontology to represent the semantic of Library Search. we improve Library and courses Search for E-learning Content Management System using mobile agent based on Semantic web technologies such as Resource Description Framework (RDF), RDF Schema (RDFS), Web Ontology Language (OWL), Uniform Resource Identifier (URI), XML, and SPARQ. We apply the training at Damietta port in Egypt as a realworld case study. It may advise a learner with most suitable learning objects. we present one of possible applications of mobile agent technology based on semantic web to management of Web Services, this model improve the information retrieval and E-learning system.

The limitations of the conventional Web and how the Semantic Web overcomes those limitations have been discussed. Then we discussed the common technologies used to construct the Semantic Web. We use semantic web and mobile agent to improve Library and courses Search.

The main purpose of our paper was to illustrate possibilities of using the Semantic Web as a backbone for E-Learning.and provides flexible and personalized access to the learning materials. 
International Journal of Web \& Semantic Technology (IJWesT) Vol.6, No.3, July 2015

The main contribution is our model for Improve information retrieval and e-learning system using mobile agent based on semantic web technology, to help user to Minimal service execution time, Guaranteed, permanent connection with service, and Guaranty of confidentiality and secure private information exchange The Used Technologies are introduced

\section{Acknowledgement}

The authors would like to thank Prof. Dr. Eraky Khalifa, for his leadership, continued help, support, and encouragement. We are grateful to him for sharing his experienc, knowledge, and expertise.

\section{REFERENCES}

[1] D. F, H. Ian, V. Frank, M. Deborah and F. Peter, ""OIL: Ontology Infrastructure to Enable the Semantic Web,” IEEE Intelligent Systems, vol. 16, no. 2, 2001.

[2] ,. O. Lassila, "Web Metadata: A Matter of Semantics.," IEEE Internet Computing, vol. 2, no. 4, pp. 30-37, 1998.

[3] J. Heflin, "Toward the Semantic Web: Knowledge Representation in A dynamic, Distributed Environment," Ph.D. Thesis, University of Maryland, College Park, 2001.

[4] S. Decker, S. Melnik, F. Harmelen and D. Fensel, "The Semantic Web: The Roles of XML and RDF," IEEE internet computing, vol. 4, no. 5, p. 63, 2000.

[5] . T. David and . W. Johanna, "Web semantics and ontology ISBN: 1-59140-905-5," Idea Group, 2006.

[6] Thomas B, "Explorer's Guide to the Semantic Web," Manning, ISBN 1-932394-20-6, 2004.

[7] "Semantic_Web," 2013. [Online]. Available: http://en.wikipedia.org/wiki/Semantic_Web. [Accessed 2013].

[8] S. Aghaei, M. A. Nematbakhsh and H. K. Farsani, "EVOLUTION OF THE WORLD WIDE WEB : FROM WEB 1.0TO WEB 4.0," International Journal of Web \& Semantic Technology (IJWesT), vol. 1, p. 3, anuary 2012.

[9] P. P. I. Horrocks, “"'Three Theses of Representation in the Semantic Web,", in Proceedings of the 12th international conference on World Wide Web,, 2003.

[10] L. Yu., Introduction to the Semantic Web and Semantic Web Services, Taylor \& Francis Group, LLC, , 2007..

[11] G. Ross, "An introduction to Tim Berners-Lee's Semantic," 31 January 2005. [Online]. Available: http://www.techrepublic.com/article/an-introduction-to-tim-berners-lees-semantic-web/5552998. [Accessed 14 2013].

[12] T. Berners-Lee, "Semantic Web Road map," 1998. [Online]. Available: http://www.w3.org/DesignIssues/Semantic.html..

[13] T. Berners-Lee, J. Hendler and O. Lassila, "The Semantic Web: A new form of Web content that ismeaningful to computers will unleash a revolution of new possibilities.," Scientific American., 2001.

[14] B. Ö. Czerkawski, "THE SEMANTIC WEB IN TEACHER EDUCATION," TOJET: The Turkish Online Journal of Educational Technology, vol. 13, no. 4, October 2014.

[15] M.-B. T, Everything Integrated: A Framework for Associative Writing in the Web, University of Southampton, 2004.

[16] L. O. K. T. S. Michael Daconta, The Semantic web: the guide to future of XML, web services and knowledge management.

[17] A. B. A. J. v. d. M. A. Gerber, "Functional Semantic Web Architecture,"," Springer Berlin / Heidelberg, Lecture Notes in Computer Science, Vols. Volume 5021/2008, ISBN:978-3-540-682332, pp. pages: 273-287, 2008. 
International Journal of Web \& Semantic Technology (IJWesT) Vol.6, No.3, July 2015

[18] A. B. A. J. v. d. M. A. Gerber, "Towards a Semantic Web Layered Architecture," in Software Engineering Proceedings of the 25th conference on IASTED International Multi-Conference, Innsbruck, Austria, , 2007.

[19] M. A. a. H. S. H. Al-Feel, "Toward an Agreement on Semantic Web Architecture," in World Academy of Science,Engineering and Technology, 2009.

[20] H. Pascal and J. Krzysztof, "Semantic Web Tools and Systems," Semantic Web, vol. 2, no. 1/2011, 2011.

[21] B. P. P. P.-S. J. I. Horrocks, “Semantic Web Architecture: Stack or Two Towers?,,", Lecture Notes in Computer Science, Vols. Volume 3703/2005,, no. ISBN: 978-3-540-, pp. Pages: 37-41,, 2005.

[22] A. Swartz, "The Semantic Web in Breadth," 2002. [Online]. Available: http://logicerror.com/semanticWeb-long..

[23] R. Pandey and S. Dwivedi, "Interoperability between Semantic Web Layers: A Communicating Agent Approach," International Journal of Computer Applications, vol. 12, no. 3, p. 0975 - 8887 , November 2010.

[24] “XML_schema," [Online]. Available: http://en.wikipedia.org/wiki/XML_schema.

[25] T. Bray, "Extensible Markup Language (XML) 1.1 (Second Edition)," 2006. [Online]. Available: http://www.w3.org/TR/xml11/\#sec-origin-goals.

[26] "REC-xml-names," [Online]. Available: http://www.w3.org/TR/REC-xml-names.

[27] D. Beckett, "RDF/XML Syntax Specification (Revised)," 2004. [Online]. Available: http://www.w3.org/TR/REC-rdf-syntax/. [Accessed 2013].

[28] E. Miller, "An Introduction to the Resource Description Framework," D-Lib Magazine, 1998.

[29] "Resource Description [Online]. Avamework," Available: http://en.wikipedia.org/wiki/Resource_Description_Framework.

[30] "rdf-schema," [Online]. Available: http://www.w3.org/TR/rdf-schema.

[31] M. Obitko., "Introduction to Ontologies and Semantic Web," 2007. [Online]. Available: http://www.obitko.com/tutorials/ontologies-semantic-web/.

[32] S. S. A. Maedche, "Ontology Learning for the Semantic Web," IEEE Intelligent Systems, vol. 16, no. ISSN:1541-1672, pp. 72- 9, 2001.

[33] “owl-features," [Online]. Available: http://www.w3.org/TR/owl-features.

[34] "Logic layer," [Online]. Available: http://www.w3.org/2002/Talks/04-sweb/slide20-0.html.

[35] L. Stojanovic, S. Staab and R. Studer, "eLearning based on the Semantic Web," in WebNet'2001 World Conference of the WWW and Internet. AACE (2001), 2001.

[36] T. Berners-Lee, "What the Semantic Web can represent," 1709 1998. [Online]. Available: http://www.w3.org/DesignIssues/RDFnot.html. [Accessed 3103 2013].

[37] “The Semantic Web: An Introduction," [Online]. Available: http://infomesh.net/2001/swintro..

[38] T. Wilson, "'How Semantic Web Works," [Online]. Available: http://www.howstuffworks.com/semantic-web.htm.

[39] H. Zimmermann, "“'OS1 Reference Model-The IS0 Model of Architecture for Open Systems Interconnection,” IEEE Transactions on Communications, Vols. Vol. COM-28 , No. 4, 1980.

[40] S. K. M. Navneet Bhardwaj, "Meeting the challenge of various layers of Semantic Web Architecture (Research Paper)," [Online]. Available: http://www.scribd.com/doc/3336624/Meeting-the-challengeof-various-layers-of-Semantic-Web-Architecture-Research-Paper.

[41] R. O. C. Bizer, "“Using Context- and Content-Based Trust Policies on the Semantic Web," in International World Wide Web Conference, 2004.

[42] W. Nejdl, "Learning repositories - technologies and context," in ED-MEDIA 2001 World Conference on Educational Multimedia, Hypermedia \& Telecommunications,, USA, AACE., 2001. 